\title{
Effects of age on biological and functional characterization of adipose-derived stem cells from patients with end-stage liver disease
}

\author{
YANYU JIN ${ }^{1}$, LEI YANG ${ }^{2}$, YANYAN ZHANG $^{3,4}$, WEI GAO ${ }^{5}$, ZHI YAO ${ }^{6}$, \\ YANG $\mathrm{SONG}^{7}$ and YULIANG WANG ${ }^{2}$
}

Departments of ${ }^{1}$ General Surgery and ${ }^{2}$ Clinical Laboratory Medicine, Tianjin First Central Hospital, Tianjin 300192,

P.R. China; ${ }^{3}$ National Institute of Health and Medical Research, UMRS 1170, CNRS GDR 3697 Micronit;

${ }^{4}$ Laboratory of Normal and Pathological Hematopoiesis, Gustave Roussy Institute, University Paris-Sud, University of Saclay, 94805 Villejuif, France; ${ }^{5}$ Organ Transplant Center, Tianjin First Central Hospital,

Tianjin 300192; ${ }^{6}$ Department of Immunology, Tianjin Medical University, Tianjin 300070;

${ }^{7}$ Department of Neurology, Tianjin First Central Hospital, Tianjin 300192, P.R. China

Received March 2, 2017; Accepted May 22, 2017

DOI: $10.3892 / \mathrm{mmr} .2017 .6967$

\begin{abstract}
Adipose-derived mesenchymal stromal cells (ADSCs) possess a multilineage potential and immunoregulatory properties, and may have great potential in autologous cell-based technologies. The aim of the present study was to investigate how the age of patients with benign end-stage liver disease affected the biological and functional characteristics of ADSCs, which is important for increasing the potential effectiveness of autologous cell therapy. ADSCs were obtained and cultured from three distinct age groups: Infant, adult and elderly. Cell immunophenotypic characteristics and antiapoptotic capacity were determined by flow cytometry, and cell proliferation and migration were monitored with a Real-Time Cell Analyzer. Multilineage differentiation potential was investigated by evaluating the induction response and by reverse transcription-quantitative polymerase chain reaction. Suppression of T cell proliferation was assessed in a co-culture system by MTT assay. The regulatory $\mathrm{T}$ cells (Tregs) were analyzed by flow cytometry, and ELISAs were performed to detect the cytokine profile in culture supernatants. All ADSC sample phenotypes were characterized as $\mathrm{CD}{ }^{+} / \mathrm{CD} 3^{+} / \mathrm{CD} 105^{+} / \mathrm{CD} 45^{-} / \mathrm{CD} 34^{-}$, and the apoptotic rate was not statistically different among all ages. However, the proliferation and migratory capacity were significantly increased in infant-derived ADSCs. In addition, ADSCs derived from infant patients demonstrated a relatively high
\end{abstract}

Correspondence to: Professor Yuliang Wang, Department of Clinical Laboratory Medicine, Tianjin First Central Hospital, 24 Fukang Road, Nankai, Tianjin 300192, P.R. China

E-mail: wang_yu_1@163.com

Key words: benign end-stage liver disease, adipose tissue, mesenchymal stromal cells, immunomodulation, infant proclivity for osteogenic differentiation compared with cells derived from either adult or elderly patients. Furthermore, ADSCs co-cultured with mitogen-activated T cells significantly suppressed T-cell proliferation, downregulated the secretion of interferon- $\gamma$ and increased the percentage of Tregs, with infant-derived ADSCs being most effective. Results from the present study indicated that ADSCs derived from infant patients may have biological advantages compared with older cell sources, and may provide an effective reference for the clinical application of ADSCs.

\section{Introduction}

Liver fibrosis is characterized as reduced liver regeneration and liver dysfunction, and may lead to portal hypertension and end-stage liver disease (ESLD) (1). Currently, liver transplantation (LT) is the only option that may improve the survival rate of adult and pediatric patients with ESLD; however, the severe shortage of donor organs limits the widespread application of LT $(2,3)$. This problem has been partially alleviated by reduced-size LT (RSLT), such as living donor LT and split LT (4). However, unavoidable and potentially serious ischemia/reperfusion injuries that are associated with RSLT have been reported to impair the regeneration of remnant liver tissue (5). Therefore, effective therapeutic strategies aimed at inhibiting hepatocyte apoptosis/necrosis and stimulating the regeneration would be beneficial for patients on LT waiting lists, as they would offer more time and increase the chances of obtaining LT, as well as for patients with small-for-size syndrome following living or split LT.

Our increasing knowledge on stem cell biology has offered new opportunities for stem-cell-based therapies as a platform in regenerative medicine for liver diseases (6,7). Mesenchymal stromal cells (MSCs) are considered a promising tool for cell-based therapy owing to their extensive self-renewal and multidifferential potency, as well as their ability to stimulate blood vessel and nerve growth, and particularly their 
production of cytokines and growth factors $(8,9)$. Recently, MSCs derived from bone marrow, adipose tissue or umbilical cord blood have been successfully applied for the treatment of ESLD (10-12), and patients with ESLD exhibited good tolerance and decreased Model for End-stage Liver Disease scores, as well as improvements in albumin production and liver function follwing 6-months follow-up. In addition, MSC-based therapy may be an effective auxiliary therapy for RSLT (13).

Although bone marrow is the most common and well-characterized source of MSCs, adipose tissue is being recognized as a promising source suitable for autologous stem-cell therapy. Adipose tissue is one of the richest sources for MSCs, and they are easily accessible through less invasive and safe collection procedures, and they maybe the best choice for autologous cell transplantation (14). Our previous study demonstrated that autologous adipose-derived MSCs (ADSCs) transplantation resulted in enhanced liver regeneration capacity of the remnant liver tissue in the early phase following repeat partial hepatectomy (15). Although this result was considered promising and encouraging, the therapeutic efficacy of autologous MSCs for most clinical applications remains modest, possibly due to the decreased regenerative potential in aged patients with chronic diseases (16). To effectively understand the properties of ADSCs affected by age and disease, autologous clinical applications are required. To the best of our knowledge, human infantile ADSCs have not yet been compared with adult and elderly cell populations with benign ESLD. Accordingly, the aim of the present study was to investigate how the age of patients with benign ESLD affected the biological and functional characteristics of ADSCs, which is crucial to increasing the potential effectiveness of autologous cell therapy.

\section{Materials and methods}

Patients. A total of 30 male patients who underwent living-donor LT for benign ESLD, between January and December 2014, in Tianjin First Central Hospital (Tianjin, China), were enrolled in the present study. During the LT procedure, samples of abdominal subcutaneous adipose tissue were obtained from three distinct age groups: i) Infant, $<1$ year old ( $n=10)$; ii) adult, 20-40 years old $(n=10)$; and iii) elderly, $>50$ years old $(n=10)$. Samples were immediately transported to the Department of Clinical Laboratory Medicine, Tianjin First Central Hospital.

The present study was authorized by the Ministry of Health (Beijing, China). Following approval by the Medical Ethics Committee of Tianjin First Center Hospital, all patients signed a written, informed consent, in accordance with the Institutional Review Board guidelines for the protection of human patients. In cases involving infants, informed consent was obtained from their parents or legal guardians.

Preparation and characterization of ADSCs. Isolation and culture of ADSCs was performed as previously described (17). Briefly, isolated adipose tissues were minced and digested with type I collagenase (Sigma-Aldrich; Merck KGaA, Darmstadt, Germany) for $30 \mathrm{~min}$ at $37^{\circ} \mathrm{C}$ with shaking. The mixture was washed, centrifuged at $300 \mathrm{x} \mathrm{g}$ for $5 \mathrm{~min}$ at $37^{\circ} \mathrm{C}$ and resuspended in Dulbecco's modified Eagle's medium/Ham's F12 (DMEM/F12; Gibco; Thermo Fisher Scientific, Inc.,
Waltham, MA, USA), and sieved through a Falcon mesh cell strainer (70 $\mu \mathrm{m}$; BD Biosciences, Franklin Lakes, NJ, USA) to eliminate the undigested fragments. Following centrifugation at $300 \mathrm{x} \mathrm{g}$ for $5 \mathrm{~min}$ at $37^{\circ} \mathrm{C}$, ADSCs were resuspended and cultured in DMEM/F12 containing 10\% fetal bovine serum (FBS; Gibco; Thermo Fisher Scientific, Inc.) at $37^{\circ} \mathrm{C}$, $5 \% \mathrm{CO}_{2}$ and $95 \%$ humidity. Cells were grown to $80 \%$ confluence, and adherent cells were detached with trypsin/EDTA (Sigma-Aldrich; Merck KGaA) and used in subsequent experiments at the third passage. Cultured ADSCs were blocked in $10 \%$ human AB serum (Sigma-Aldrich; Merck KGaA), then incubated with fluorescein isothiocyanate-conjugated CD45 (cat no. 555482), CD73 (cat no. 561254), CD90 (cat no. 55595), CD34 (cat no. 555821) and CD105 (cat no. 562408), all obtained from BD Biosciences, for $40 \mathrm{~min}$ at $4^{\circ} \mathrm{C}$ at a dilution of 1:50. After adding $2 \mathrm{ml}$ PBS, cells were centrifuged at $150 \mathrm{x} \mathrm{g}$ for $5 \mathrm{~min}$ at $8^{\circ} \mathrm{C}$. Then data were acquired using a flow cytometer (FACSCalibur), and data were analyzed using the CellQuest 6.0 software (both from BD Biosciences) (18). The differentiation capacity of ADSCs into adipogenic and osteogenic lineages was analyzed as previously described (18). The ADSCs were seeded in DMEM/F12 at $2 \times 10^{4}$ cells $/ \mathrm{cm}^{2}$ in six-well tissue culture plates. When the cells reached $100 \%$ confluency, DMEM/F12 was subsequently replaced with specific inducer medium. Adipogenic inducer medium was DMEM/F12 containing $1 \mu \mathrm{mol} / 1$ dexamethasone, $0.5 \mathrm{mmol} / 13$-isobutyl-1-methylxanthine, $5 \mathrm{mg} / 1$ insulin and $100 \mu \mathrm{mol} / 1$ indomethacin (all Sigma-Aldrich; Merck KGaA). Osteogenic inducer medium was DMEM/F12 containing $100 \mathrm{nmol} / 1$ dexamethasone, $10 \mathrm{mmol} / \mathrm{l} \beta$-sodium glycerophosphate and $50 \mu \mathrm{g} / \mathrm{ml}$ vitamin $\mathrm{C}$ (all Sigma-Aldrich; Merck KGaA). Following a 21-day induction period, adipocytes and osteoblasts were identified by Oil Red O staining (Sigma-Aldrich; Merck KGaA) and Alizarin red S staining (Genmed Gene Pharmaceutical Technology, Co., Ltd., Shanghai, China) at room temperature for $10 \mathrm{~min}$, respectively. Cells were visualized under an inverted Olympus IX71 microscope at x200 magnification and photomicrographs were recorded using UIS2 software (both from Olympus Corporation, Tokyo, Japan).

Cell proliferation assay. Cell proliferation was monitored with the highly sensitive xCELLigence Real-Time Cell Analyzer (RTCA; ACEA Biosciences, Inc., San Diego, CA, USA), which is able to measure the status of cell growth in real time. Briefly, ADSCs were incubated in $60 \mathrm{~mm}$ culture plates for $24 \mathrm{~h}$, and 5,000 cells in $100 \mu \mathrm{l}$ of culture medium were subsequently seeded into each sensor-containing well of an E-Plate 16 microtiter plate (Roche Diagnostics, Indianapolis, IN, USA), which was locked into the RTCA device and incubated at $37^{\circ} \mathrm{C}$ with $5 \% \mathrm{CO}_{2}$ for $72 \mathrm{~h}$. While seeded cells were propagated in the incubator, impedance values were converted into cell index (CI) value corresponding to each well. CI value is defined as relative change in measured electrical impedance to represent cellular proliferation status. CI was read automatically every $15 \mathrm{~min}$ for $72 \mathrm{~h}$. All experiments were carried out in triplicate. Data were analyzed using xCELLigence software, version 1.2 (RTCA; ACEA Biosciences, Inc.).

Cell migration assay. Cell migration was monitored in real-time using the RTCA system (ACEA Biosciences, Inc.) 
and expressed as the cell migration index (CMI). The RTCA platform is able to measure cell migration status in real time. Following overnight starvation of $1 \times 10^{5}$ cells in DMEM/F12 medium with $0.2 \% \mathrm{FBS}, 1 \times 10^{4}$ cells in $100 \mu \mathrm{l}$ serum-free medium were seeded in the upper chamber of a CIM-Plate 16 (Roche Diagnostics), and the lower chamber was filled with $160 \mu \mathrm{l}$ DMEM/F12 medium containing 20\% FBS. The CIM plate was placed in the RTCA device and incubated for $72 \mathrm{~h}$ at $37^{\circ} \mathrm{C}$ in a $5 \% \mathrm{CO}_{2}$ atmosphere. A reading of the impedance on the underside was taken every $10 \mathrm{~min}$ and reported as a CII, which was derived from the relative change in electrical impedance set against the baseline reading that was taken with no cells and only DMEM/F12 medium. All experiments were performed in triplicate. Data were analyzed using $\mathrm{xCELLigence} \mathrm{software.}$

Determination of ADSCs antiapoptotic ability. Apoptotic cell death was evaluated using Annexin V-fluorescein isothiocyanate (FITC)/propidium iodide (PI) Apoptotic Detection kit (BD Biosciences) and flow cytometry, according to the manufacturer's protocol. To compare the antiapoptotic ability of the three ADSC groups, passage 3 cells were trypsinized, adjusted to a concentration of $3 \times 10^{5}$ cells $/ \mathrm{ml}$ and seeded into 6 -well plates. ADSCs were grown to $80 \%$ confluence, dexamethasone $\left(1 \times 10^{-6} \mathrm{~mol} / \mathrm{l}\right)$ was added and incubated at $37^{\circ} \mathrm{C}$ with $5 \% \mathrm{CO}_{2}$ and $95 \%$ humidity for $48 \mathrm{~h}$. Following incubation, cells were collected by trypsinization, and centrifuged at $150 \mathrm{x}$ g for $5 \mathrm{~min}$ at $8^{\circ} \mathrm{C}$, and suspended in binding buffer (BD Biosciences). Annexin V-FITC/PI was added and the cells were incubated in the dark at room temperature for $15 \mathrm{~min}$ prior to analyzing using a flow cytometer (FACSCalibur), and data were analyzed using the CellQuest 6.0 software (both from BD Biosciences).

Reverse transcription-quantitative reverse transcription polymerase chain reaction $(R T-q P C R)$. mRNA expression levels of the osteogenic-specific gene osteopontin (OPN) and the adipogenic-specific gene peroxisome proliferator-activated receptor- $\gamma($ PPAR- $\gamma)$ were detected by RT-qPCR. Briefly, total RNA was extracted from $1 \times 10^{6}$ ADSCs using TRIzol Reagent (Invitrogen; Thermo Fisher Scientific, Inc.) and reverse transcribed into cDNA using PrimeScript RT Reagent kit (Takara Biotechnology Co., Ltd., Dalian, China), according to the respective manufacturer's protocols. RNA purity was verified by an optical density (OD)260/OD280 $\mathrm{nm}$ absorption ratio $>1.9$. qPCR was performed using a LightCycler 480 system (Roche Diagnostics, Basel, Switzerland) and SYBR Premix Ex Taq (Takara Biotechnology Co., Ltd.). Sequences of the primers used were as follows: PPAR- $\gamma$ forward, 5'-AGTCCTCACAGC TGTTTGCCAAGC-3' and reverse, 5'-GAGCGGGTGAAG ACTCATGTCVTGTC-3'; OPN forward, 5'-AAACGCCGA CCAAGGTACAG-3' and reverse, 5'-ATGCCTAGGAGG CAA AAGCAA-3'; GAPDH forward, 5'-AGAAGGCTGGGGCTC ATTTG-3' and reverse, 5'-AGGGGCCATCCACAGTCTTC-3'. The PCR cycling conditions were a hot-start step of $95^{\circ} \mathrm{C}$ for $10 \mathrm{~min}$, followed by a 40 -cycle program including a denaturation phase at $95^{\circ} \mathrm{C}$ for $30 \mathrm{sec}$, annealing at $60^{\circ} \mathrm{C}$ for $45 \mathrm{sec}$ and exten sion for $30 \mathrm{sec}$ at $72^{\circ} \mathrm{C}$. Data were analyzed using the LightCycler 480 software version 1.5 (Roche Diagnostics). Relative gene expression analysis was calculated relative to the housekeeping gene GAPDH (19). The assay was replicated in 3 independent experiments.

Co-culture of ADSCs and peripheral blood mononuclear cells (PBMCs). PBMCs were isolated from heparinized peripheral blood samples of the healthy volunteers using Ficoll-Hypaque (Sigma-Aldrich; Merck KGaA) density gradient centrifugation at $400 \mathrm{x} \mathrm{g}$ for $25 \mathrm{~min}$ at room temperature, after receiving informed consent. The immunosuppressive capacity of ADSCs was tested in a co-culture of ADSCs with PBMCs as follows. A total of $1.0 \times 10^{6}$ cells were mitotically inactivated with mitomycin C (50 $\mu \mathrm{g} / \mathrm{ml}$; Sigma-Aldrich; Merck KGaA) for $30 \mathrm{~min}$ at $37^{\circ} \mathrm{C}$ in serum-free medium. ADSCs were subsequently washed 3 times with PBS and seeded into a 96-well $\left(5.0 \times 10^{3}\right.$ cells/well $)$ or 6 -well $\left(5.0 \times 10^{4}\right.$ cells/well $)$ flat-bottomed plate (Nalge Nunc International; Thermo Fisher Scientific, Inc.) for proliferation or for flow cytometry, respectively, in triplicate. Following overnight adherence at $37^{\circ} \mathrm{C}$, PBMCs $\left(5.0 \times 10^{4}\right.$ cells/well in the 96 -well plates; $5.0 \times 10^{5}$ cells/well in the 6-well plates) were seeded onto the cultured ADSCs at a ratio of 1:10 (ADSC:PBMSC), stimulated with phytohemagglutinin (PHA; $5 \mu \mathrm{g} / \mathrm{ml}$; Sigma-Aldrich; Merck KGaA) and interleukin 2 (IL-2; $5 \mathrm{ng} / \mathrm{ml}$; R\&D Systems, Minneapolis, MN, USA) to activate T-cell proliferation, and cultured at $37^{\circ} \mathrm{C}$ in a humidified atmosphere of $5 \% \mathrm{CO}_{2}$. PBMCs cultured alone with PHA and IL-2 served as a control. Following culture for 3 days, PBMCs were collected via pipetting for T-cell proliferation assay and flow cytometric analysis. Supernatants from the cell cultures were collected and stored at $-80^{\circ} \mathrm{C}$ for subsequent analysis of cytokine production.

Suppression of T-cell proliferation. MTT colorimetry was used to measure T-cell proliferation. Briefly, following removal of the PBMCs to a new 96-well plate, $5 \mathrm{mg} / \mathrm{ml} \mathrm{MTT} \mathrm{solution}$ (Sigma-Aldrich; Merck KGaA) was added to each well and the plate was incubated for $4 \mathrm{~h}$ at $37^{\circ} \mathrm{C}$. Subsequent to the incubation, $100 \mu$ ldimethyl sulfoxide (Sigma-Aldrich; Merck KGaA) was added to terminate the reactions, and the plates were placed on a shaker $(150 \mathrm{rpm})$ for $10 \mathrm{~min}$ to fully dissolve the crystals. The absorbance value at $570 \mathrm{~nm}$ was measured using an automatic BioTek Synergy 2 Multi-Mode microplate reader (BioTek Instruments, Inc., Winooski, VT, USA), with each sample assayed in triplicate. Data were analyzed using the Gen5 version 3.03 data analysis software (BioTek Instruments, Inc.). The proliferation inhibitory rate was calculated according to the formula: Inhibition rate $(\%)=[1-$ (average absorbance for experimental group/average absorbance for control group)] x100.

Flow cytometric assessment of $\mathrm{CD}^{+}$regulatory $\mathrm{T}$ cells (Tregs). For examination of $\mathrm{CD}^{+} / \mathrm{CD} 25^{+} /$forkhead box P3 $\left(\right.$ FoxP3) ${ }^{+}$Tregs, monoclonal antibodies specific to CD4-FITC (cat no. 555346) and CD25-allophycocyanin (cat no. 555434) surface staining at a dilution of 1:50 was conducted in co-cultured and harvested PBMCs. Following the incubation for $20 \mathrm{~min}$ in the dark at $37^{\circ} \mathrm{C}$, the cells were washed with PBS, and FoxP3-phycoerythrin (cat no. 560046) (all from BD Biosciences) intracellular staining was conducted and incubation for $30 \mathrm{~min}$ in the dark at $37^{\circ} \mathrm{C}$ followed, as previously described (20). Data were acquired using a flow 

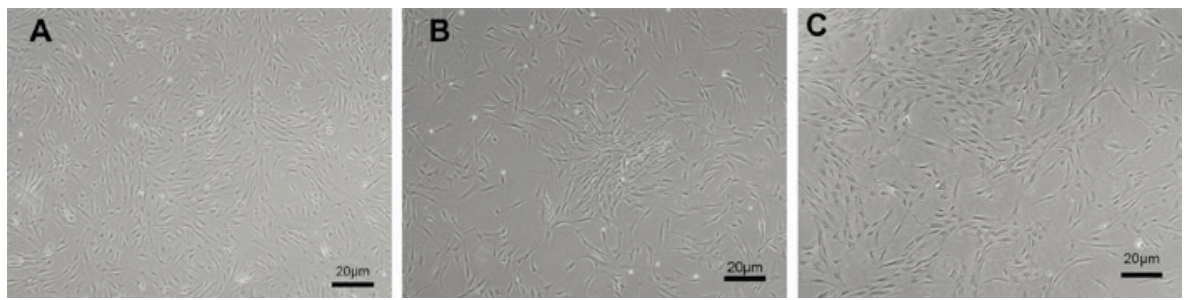

Figure 1. Morphologic characteristics of isolated ADSCs from three age groups. (A) Infant-derived ADSCs. (B) Adult-derived ADSCs. (C) Elderly-derived ADSCs. Magnification, x20. ADSCs, adipose-derived mesenchymal stromal cells.
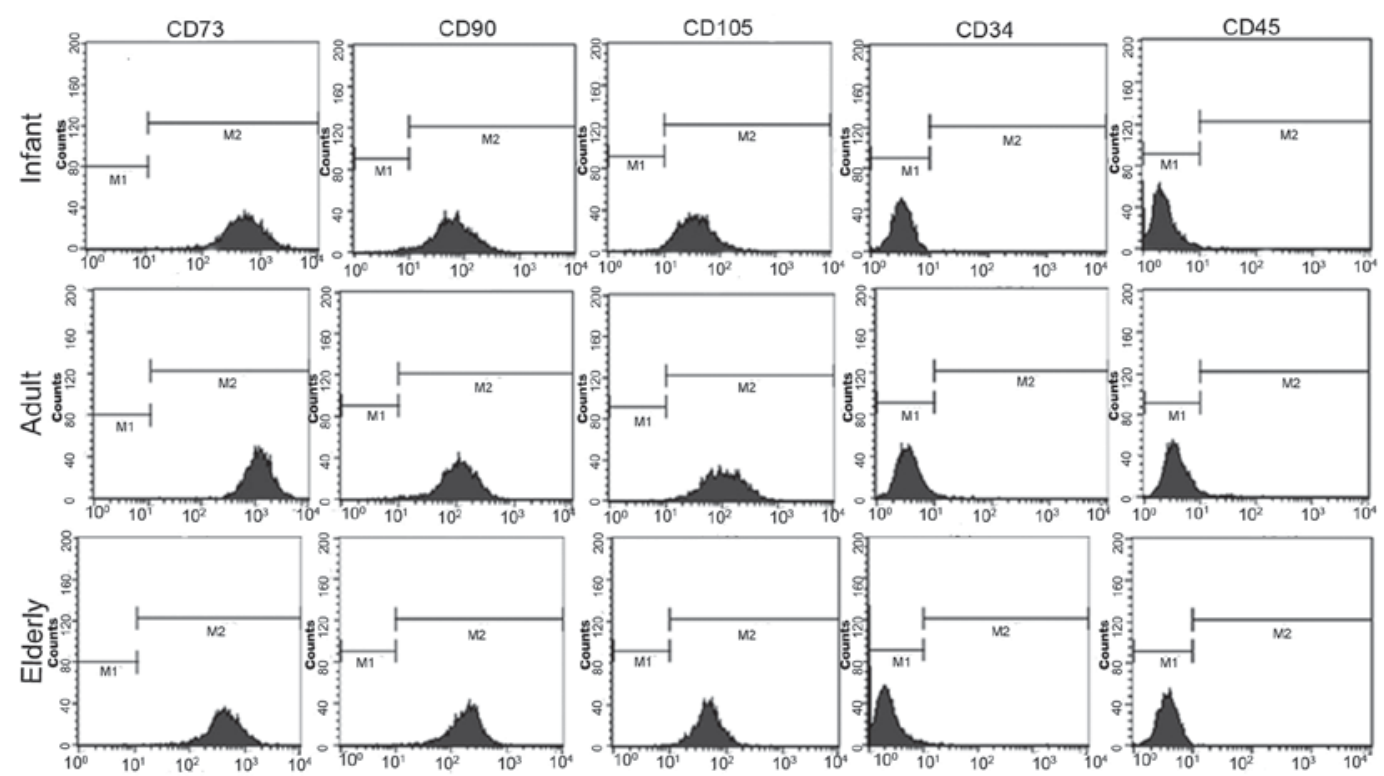

Figure 2. Flow cytometric analysis of immunophenotypic characteristics of isolated ADSCs derived from infant, adult and elderly patients. In each group, ADSCs were positive for CD73, CD90 and CD105, and negative for CD34 and CD45. ADSCs, adipose-derived mesenchymal stromal cells.

cytometer (FACSCalibur flow cytometer; Becton-Dickinson) and data were analyzed using the CellQuest 6.0 software (BD Biosciences).

Cytokine production. Interferon (IFN)- $\gamma$ and interleukin (IL)-10 concentrations in the supernatants of the T cell proliferation co-culture aforementioned were measured using a commercially available IFN- $\gamma$ (cat no. BMS228) and IL-10 (cat no. BMS215) human ELISA kits (eBioscience; Thermo Fisher Scientific, Inc.), according to the manufacturer's instructions.

Statistical analysis. Data are expressed as the mean \pm standard deviation. Statistical analysis was performed using one-way analysis of variance followed by a post-hoc Bonferroni multiple comparison test. Statistical analyses were performed using SPSS 16.0 software (SPSS, Inc., Chicago, IL, USA). P<0.05 was considered to indicate a statistically significant difference.

\section{Results}

ADSC morphology and immunophenotype is independent of patient age. ADSCs were successfully isolated from abdominal subcutaneous adipose tissues from all patients within each of the three groups of LT recipients (n=10/group), which spanned a wide range of ages (6 months to 60 years). Homogeneous populations of ADSCs were cultured to $80 \%$ confluence; ADSCs derived from infant, adult and elderly patients exhibited fibroblast-like morphology and a whirlpool-like or parallel array (Fig. 1). In addition, ADSCs derived from all age groups exhibited positive surface antigenicity for CD73, CD90 and CD105, and exhibited negative surface antigenicity for CD34 and CD45 (Fig. 2). No notable differences were identified in relation to patient age.

Proliferative potential of ADSCs. The proliferative potential of ADSCs isolated from adipose tissue of patients with benign ESLD was monitored in real time for $72 \mathrm{~h}$. Growth curve analysis demonstrated that the proliferation CI value from infant patients was significantly increased relative to that observed in cells from both adult and elderly patients $(\mathrm{P}<0.05$; Fig. 3$)$. This difference was apparent at $48 \mathrm{~h}$ and $72 \mathrm{~h}$, which indicated a trend toward decreasing proliferative potential with age. This trend was also apparent when the proliferation CI values obtained from adult patients were compared with those obtained from elderly patients; however, no statistical significance was identified between the CI values of adult and elderly patients.

Effects of age on the migration ability of ADSCs. Migration curve analysis of ADSCs demonstrated that their CIMI values 


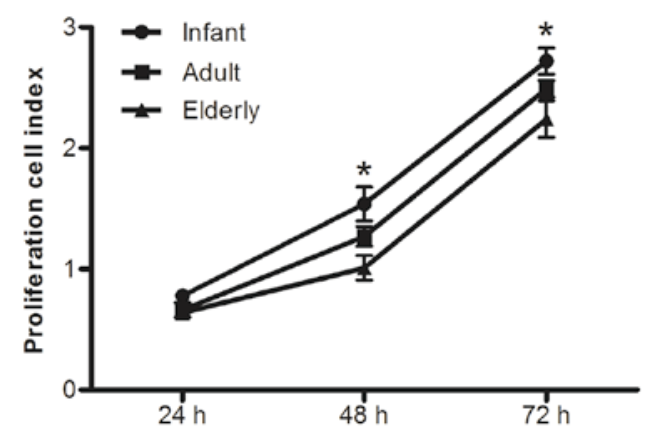

Figure 3. Growth curve analysis of isolated ADSCs derived from infant, adult and elderly patients. Proliferation cell index values of ADSCs were analyzed in real time and comparisons were made at 24,48 and $72 \mathrm{~h}$ incubation. Data are presented as the mean \pm standard deviation; " $\mathrm{P}<0.05$ vs. adult- and elderly-derived ADSCs. ADSCs, adipose-derived mesenchymal stromal cells.

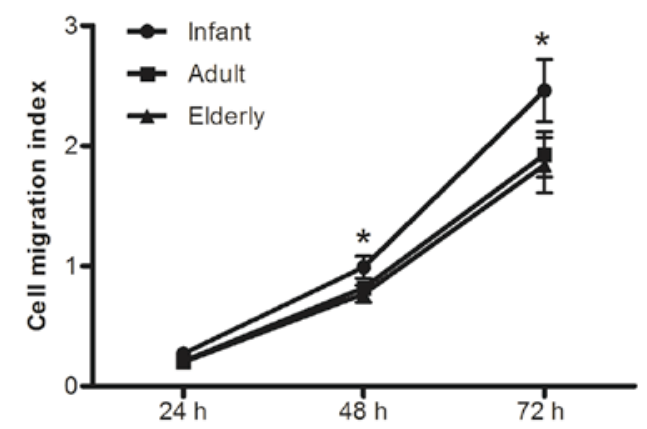

Figure 4. Migration curve analysis of isolated ADSCs derived from infant, adult and elderly patients. Cell migration index values of the ADSCs were monitored in real time, and comparisons were made at 24,48 and $72 \mathrm{~h}$ incubation. Data are presented as the mean \pm standard deviation. ${ }^{*} \mathrm{P}<0.05$ vs. adult and elderly-derived ADSCs. ADSCs, adipose-derived mesenchymal stromal cells.

were significantly increased in infants compared with adult and elderly patients $(\mathrm{P}<0.05$; Fig. 4$)$. This difference was apparent at $48 \mathrm{~h}$ and $72 \mathrm{~h}$, which indicated a trend towards decreasing cell migratory ability with age. Adult-derived ADSCs exhibited slightly higher migration capability compared with elderly-derived ADSCs; however no statistically significant difference was identified between the two groups $(\mathrm{P}>0.05)$.

Antiapoptotic ability of ADSCs. Flow cytometric results indicated that no statistically significant differences were identified among the three age groups in antiapoptotic capacity induced by high concentration of dexamethasone (Fig. 5).

Adipogenic and osteogenic capability of ADSCs. The adipogenic and osteogenic differentiation potential of ADSCs derived from three age groups were evaluated by comparing the amount of lipid-rich vacuoles and calcium deposition produced in medium only (Fig. 6). ADSCs were able to efficiently differentiate toward the adipogenic and osteogenic lineages. Oil Red O staining for adipocytic-differentiated cells indicated no notable difference in infant vs. adult or elderly-derived ADSCs. Alizarin Red S staining for osteogenic-differentiated cells was notably stronger in the infant-derived ADSCs, which indicated that infant ADSCs produced a greater amount of

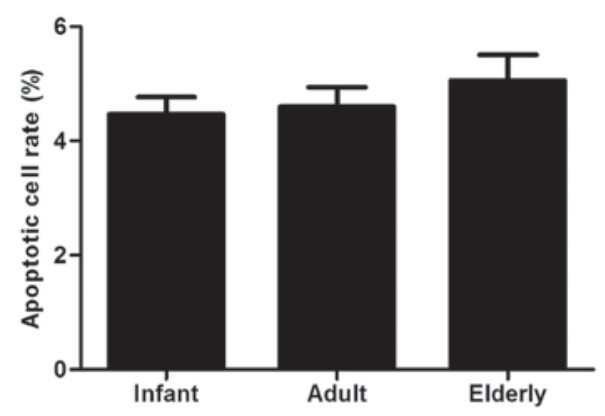

Figure 5. Flow cytometric analysis of antiapoptotic ability of isolated ADSCs derived from infant, adult and elderly patients. Data are presented as the average \pm standard deviation. ADSCs, adipose-derived mesenchymal stromal cells.

calcium deposition compared with cells derived from adult and elderly patients.

Adipogenic and osteogenic inductions were further evaluated by RT-qPCR analysis of the lineage-specific genes PPAR $-\gamma$ (adipocytes) and OPN (osteocytes). The mRNA expression levels of PPAR- $\gamma$ appeared to gradually increase with patient age (Fig. 7A); however, this difference was not identified as statistically significant, which indicated that age may not affect adipogenic capacity. Conversely, significant age-related differences were identified in the OPN mRNA expression levels of ADSCs isolated from the three age groups, with the highest level in infant-derived ADSCs $(\mathrm{P}<0.01)$ and a notably increased level in adult-derived ADSCs compared with elderly-derived ADSCs ( $\mathrm{P}<0.05$; Fig. 7B).

Suppression of T-cell proliferation. The effects of biological aging on ADSC-mediated suppression of T-cell proliferation was examined following co-culture with PBMCs. ADSCs were demonstrated to strongly inhibit the proliferation of $\mathrm{T}$ cells in response to PHA and IL-2 stimulation. The adult- and elderly-derived ADSCs were significantly less efficient at suppressing of T-cell proliferation compared with infant-derived ADSCs ( $\mathrm{P}<0.01$; Fig. 8), indicating an age-associated decline in the immunomodulatory capacity ADSCs.

$\mathrm{CD}^{+} / \mathrm{CD} 25^{+} / \mathrm{FoxP}^{+}$Treg analysis. ADSCs from each of the three groups were revealed to significantly increase the proportion of $\mathrm{CD}^{+} / \mathrm{CD} 25^{+} / \mathrm{FoxP} 3^{+}$Tregs when co-cultured with PBMCs, compared with PBMCs alone ( $\mathrm{P}<0.01$; Fig. 9). The proportion of $\mathrm{CD}^{+} / \mathrm{CD} 25^{+} / \mathrm{FoxP} 3^{+}$Tregs was significantly lower in adult- and elderly-derived ADSCs compared with infant-derived ADSCs $(\mathrm{P}<0.05)$.

Cytokine production. The concentrations of proinflammatory cytokine IFN- $\gamma$ were significantly decreased in the supernatants of all ADSC groups co-cultured with PBMCs, compared with the concentration in PBMCs alone ( $\mathrm{P}<0.01$; Fig. 10A). Conversely, the concentrations of anti-inflammatory cytokine IL-10 were significantly increased in the supernatants of all ADSC groups co-cultured with PBMCs compared with those in the culture of PBMCs alone ( $\mathrm{P}<0.01$; Fig. 10B). Adult- and elderly-derived ADSCs significantly increased the production of IFN- $\gamma$ and significantly decreased the production of IL-10 compared with infant-derived ADSCs $(\mathrm{P}<0.05)$. 


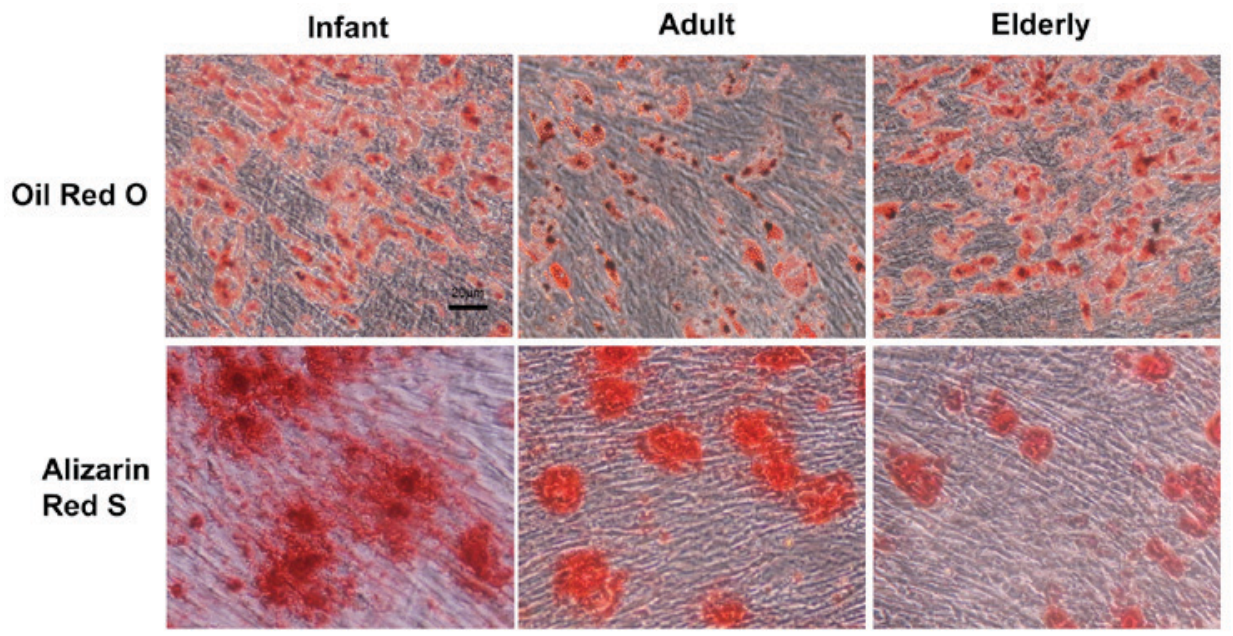

Figure 6. Multilineage differentiation potential of isolated ADSCs derived from infant, adult and elderly patients. ADSCs were fixed and stained with either Oil Red O, which indicates the formation of lipid droplets (a hallmark of adipogenesis), or Alizarin Red S, which indicates cell-mediated calcium accretion (a hallmark of osteogenesis. Magnification, x20. ADSCs, adipose-derived mesenchymal stromal cells.
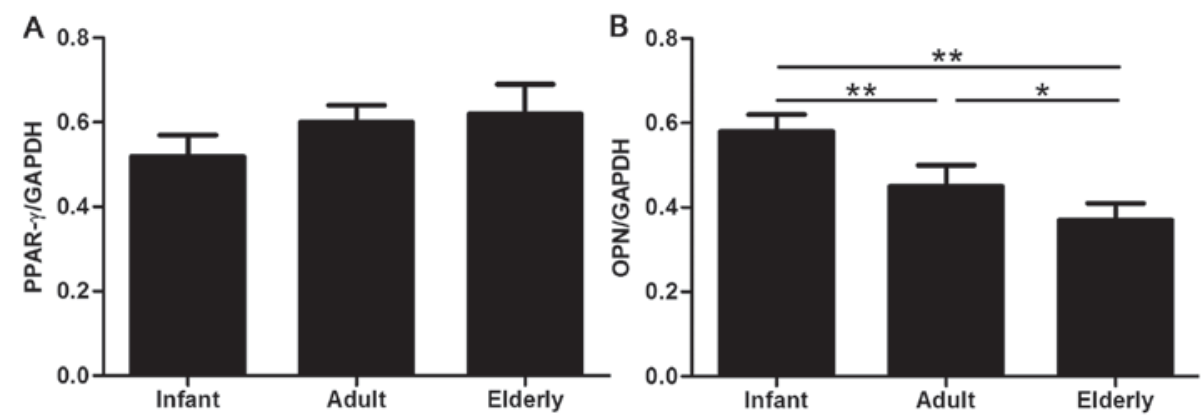

Figure 7. Reverse transcription-quantitative polymerase chain reaction analysis of adipocyte- and osteocyte-specific gene expression. (A) mRNA expression levels of the adipocyte-specific gene PPAR- $\gamma$. (B) mRNA expression levels of the osteocyte-specific gene OPN. Expression levels were normalized to GAPDH. Data are presented as the mean \pm standard deviation; ${ }^{~} \mathrm{P}<0.05 ;{ }^{* *} \mathrm{P}<0.01$. ADSCs, adipose-derived mesenchymal stromal cells; OPN, osteopontin; PPAR- $\gamma$, peroxisome proliferator-activated receptor- $\gamma$.

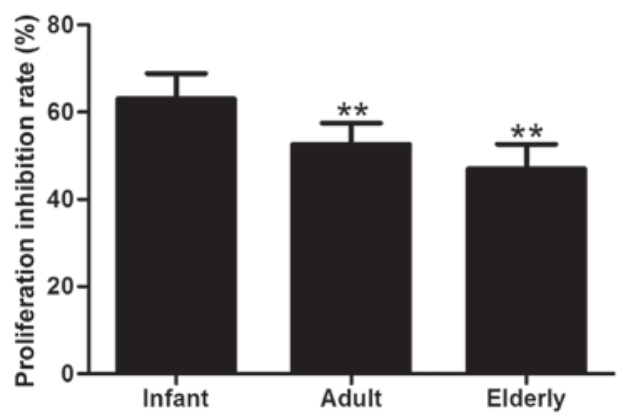

Figure 8. Proliferation inhibition rate of T cells in presence of ADSCs. PBMCs co-cultured with ADSCs from the three age groups were stimulated with phytohemagglutinin and interleukin-2. Data are presented as the mean \pm standard deviation. ${ }^{* *} \mathrm{P}<0.01$ vs. PBMCs co-cultured with infant-derived ADSCs. ADSCs adipose-derived mesenchymal stromal cells; PBMCs, peripheral blood mononuclear cells.

\section{Discussion}

A previous clinical trial involving the transendocardial administration of MSCs in patients with benign ESLD demonstrated that this technique was feasible, safe and offered potential

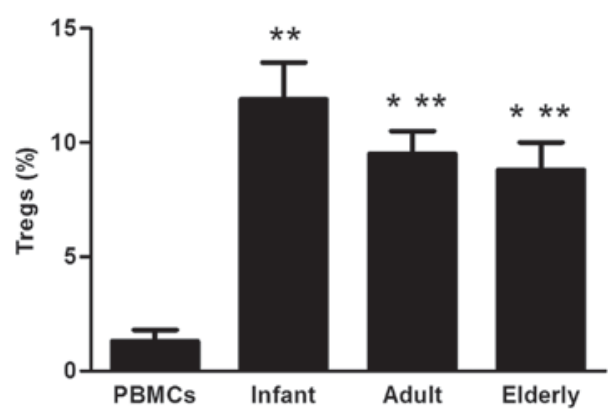

Figure 9. Induction of Tregs by ADSCs in co-culture with PBMCs. PBMCs were cultured with or without ADSCs and stimulated with phytohemagglutinin and interleukin-2. Data are presented as the mean \pm standard deviation. ${ }^{*} \mathrm{P}<0.05$ vs. PBMCs co-cultured with infant-derived ADSCs; ${ }^{* *} \mathrm{P}<0.01$ vs. PBMCs alone. ADSCs, adipose-derived mesenchymal stromal cells; PBMCs, peripheral blood mononuclear cells; Tregs, CD $4{ }^{+} \mathrm{CD} 25^{+}$Forkhead boxP $3^{+}$regulatory $\mathrm{T}$ cells.

therapeutic benefits (21). However, cellular therapeutic products such as this may be affected by aging and cell senescence, which are known to influence the properties and function of MSCs (22). Previous studies have reported adipose tissue to be an attractive autologous source of adult stem cells owing to 

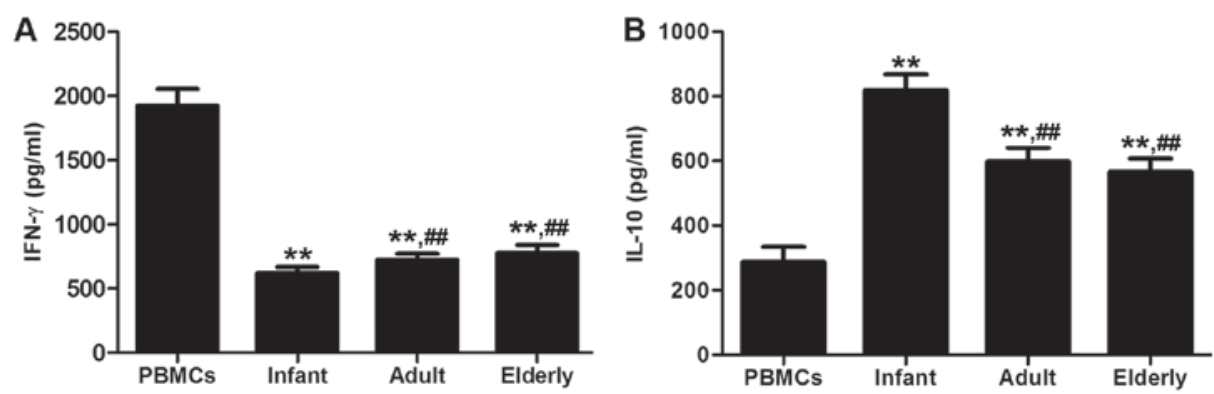

Figure 10. Cytokine profile in culture supernatants of co-cultured PBMCs and ADSCs. PBMCs were cultured with or without ADSCs and stimulated with phytohemagglutinin and interleukin-2. (A) IFN- $\gamma$ concentrations. (B) IL-10 concentrations. Data are presented as the mean \pm standard deviation. ${ }^{* * *} \mathrm{P}<0.01$ vs. PBMCs alone; ${ }^{\# \#} \mathrm{P}<0.01$ vs. PBMCs co-cultured with infant-derived ADSCs. ADSCs, adipose-derived mesenchymal stromal cells; IFN, interferon; IL, interleukin PBMCs, peripheral blood mononuclear cells.

its abundance and surgical accessibility $(23,24)$. The present study comprised a comprehensive analysis of age-associated ADSC characteristics using patients with benign ESLD, which represented a cohort of potential candidates for autologous cell therapy, and specifically elaborated on the properties of infant-derived ADSCs. The data demonstrated that increased age may be associated with reduced proliferative, migratory, differentiation and immunosuppressive capabilities and infant-derived ADSCs may have biological advantages for clinical application compared with to adult-or elderly-derived ADSCs.

The present study compared cell morphologies and immunophenotypes of ADSCs from the three patient sources. Notably, ADSCs derived from infant, adult and elderly patients exhibited typical MSC morphology and phenotype that was independent of patient age. That is, regardless of patient age, analysis of cell surface markers on ADSCs revealed the absence of expression of hematopoietic stem cells markers, such as CD34 and CD45, and an increased expression of MSC surface markers CD73, CD90 and CD105. These results were in agreement with a previous report (25), and confirm that ADSCs from all age groups contained a homogeneous phenotypic population of fibroblast-like morphology (26).

A crucial factor that may influence clinical outcomes of cell therapy/transplantation procedures, as well as the success of tissue engineering, is the proliferative, migratory, antiapoptotic and differentiation capacities of the cells used (27). The ability to expand cells without losing function is key to successfully culturing ADSCs for therapeutic purposes. In addition, ADSC migration is considered to serve a pivotal role in MSC-mediated tissue repair (28). The present study used RTCA to determine cell proliferation and cell migration, similar to a previous report (29), and demonstrated an age-related difference in cell proliferation and migration, both of which were statistically higher in infant-derived ADSCs compared with adult and elderly patients. This result suggested that the proliferation and migration of ADSCs within adipose tissue is affected by advancing age; proliferation and migration of ADSCs derived from elderly patients were further decreased, offering preliminary evidence that senescence may reduce cell proliferation and migration. However, the antiapoptotic capacity of ADSCs did not change with age (30). In addition, ASDCs derived from all patients with benign ESLD were able to efficiently differentiate into adipocytes and osteocytes in the appropriate induction environment; no significant differences were observed in the ability of ADSCs to undergo adipogenesis. However, PPAR- $\gamma$ mRNA expression analysis suggested that adipogenic capacity may increase slightly in elderly-derived ADSCs. Conversely, increased calcium deposition and increased expression of OPN mRNA in infant patients, and a comparatively lower level of OPN mRNA in elderly patients, suggested that elderly-derived ADSCs may lose their osteogenic potential and, in turn, may gain adipogenic potential, which leads to senile osteoporosis. Notably, previous studies have reported that adipogenic and osteogenic differentiation potential of ADSCs were dependent on donor age (31), whereas other studies indicated that the multilineage differentiation potential of ADSCs was not affected by age (32). This disparity may be due to the choice of age groups, group size, sex, and health or pathological states of the donors, as well as the isolation and cultivation conditions (33).

It has also been suggested that MSC therapy may improve liver function and reduce liver injury in ESLD, as supported by the notable inhibition of immunocyte proliferation and migration to the liver, an increased number of circulating Tregs, the release of soluble immunomodulatory factors and the repair of damaged target organs (34). To investigate whether ADSCs derived from patients with benign ESLD were able to effectively exert immunosuppressive properties, mitogen-activated $\mathrm{T}$ cells are co-cultured in vitro with ADSCs from the three groups of patients. Results from the present study indicated that ADSC immunomodulation may occur through a multistage process involving their ability to suppress $\mathrm{T}$ cell proliferation and convert effector $\mathrm{T}$ cells into Tregs, followed by the downregulated secretion of the proinflammatory cytokine IFN- $\gamma$ and an upregulation in anti-inflammatory cytokine IL-10 secretion. These characteristics appeared to be most effective in the infant-derived ADSCs, which indicated that there may be an age-associated decline in ADSC immunoregulatory capacity. To the best of our knowledge, this is the first study to demonstrate that infant-derived ADSCs obtained from patients with benign ESLD substantially enhanced the immunosuppressive ability of ADSCs.

Results from the present and previous studies suggested that aging may affect the properties of ADSCs and, thus, may lower the effectiveness of autologous cell therapy. For 
these reasons, cell material should be examined prior to use and the development of effective approaches for the pretreatment or modification of MSCs from elderly patients may aid in enhancing their therapeutic potential (35). Improvements may include the addition of certain growth factors and/or chemokines, such as platelet-derived growth factor and tumor necrosis factor $\alpha$, to MSC culture media, to enhance their viability, proliferation, migratory properties, which may increase their therapeutic potential $(36,37)$.

In conclusion, the present results revealed that the biological and functional characterizations of ADSCs may be positively correlated with patient age, with infant-derived ADSCs being the most effective. In addition, the results suggested that cellular pretreatment strategies may be required to improve the efficiency of aged cells in autologous therapy.

\section{Acknowledgements}

This study was supported by The National Natural Science Foundation of China (grant no. 81470982), the Tianjin Municipal Health Bureau Key Project (grant no. 16KG105) and The Tianjin Research Program of Application Foundation and Advanced Technology (grant nos. 12ZCZDSY02600 and 13JCYBJC23000).

\section{References}

1. Lindvig KP, Teisner AS, Kjeldsen J, Strøm T, Toft P, Furhmann V and Krag A: Allocation of patients with liver cirrhosis and organ failure to intensive care: Systematic review and a proposal for clinical practice. World J Gastroenterol 21: 8964-8973, 2015.

2. Reddy SS and Civan JM: From child-pugh to model for end-stage liver disease: Deciding who needs a liver transplant. Med Clin North Am 100: 449-464, 2016.

3. Lai YH, Duan WD, Yu Q, Ye S, Xiao NJ, Zhang DX, Huang ZQ, Yang ZY and Dong JH: Outcomes of liver transplantation for end-stage biliary disease: A comparative study with end-stage liver disease. World J Gastroenterol 21: 6296-6303, 2015.

4. Gong $\mathrm{N}$ and Chen X: Partial liver transplantation. Front Med 5: $1-7,2011$.

5. Raut V, Alikhanov R, Belghiti J and Uemoto S: Review of the surgical approach to prevent small-for-size syndrome in recipients after left lobe adult LDLT. Surg Today 44: 1189-1196, 2014.

6. Nicolas CT, Wang Y and Nyberg SL: Cell therapy in chronic liver disease. Curr Opin Gastroenterol 32: 189-194, 2016.

7. Than NN, Tomlinson CL, Haldar D, King AL, Moore D and Newsome PN: Clinical effectiveness of cell therapies in patients with chronic liver disease and acute-on-chronic liver failure: A systematic review protocol. Syst Rev 5: 100, 2016.

8. Malhotra S, Hu MS, Marshall CD, Leavitt T, Cheung AT, Gonzalez JG, Kaur H, Lorenz HP and Longaker MT: Mesenchymal stromal cells as cell-based therapeutics for wound healing. Stem Cells Int 2016: 4157934, 2016.

9. Prockop DJ: Inflammation, fibrosis and modulation of the process by mesenchymal stem/stromal cells. Matrix Biol 51: 7-13, 2016.

10. Suk KT, Yoon JH, Kim MY, Kim CW, Kim JK, Park H, Hwang SG, Kim DJ, Lee BS, Lee SH, et al: Transplantation with autologous bone marrow-derived mesenchymal stem cells for alcoholic cirrhosis: Phase 2 trial. Hepatology 64: 2185-2197, 2016.

11. Jang YO, Kim YJ, Baik SK, Kim MY,Eom YW, Cho MY, Park HJ, Park SY, Kim BR, Kim JW, et al: Histological improvement following administration of autologous bone marrow-derived mesenchymal stem cells for alcoholic cirrhosis: A pilot study. Liver Int 34: 33-41, 2014.

12. Salama H, Zekri AR, Medhat E, Al Alim SA, Ahmed OS, Bahnassy AA, Lotfy MM, Ahmed R and Musa S: Peripheral vein infusion of autologous mesenchymal stem cells in Egyptian HCV-positive patients with end-stage liver disease. Stem Cell Res Ther 5: 70, 2014.
13. Du Z, Wei C, Yan J, Han B, Zhang M, Peng C and Liu Y: Mesenchymal stem cells overexpressing C-X-C chemokine receptor type 4 improve early liver regeneration of small-for-size liver grafts. Liver Transpl 19: 215-225, 2013.

14. Maria AT, Maumus M, Le Quellec A, Jorgensen C, Noël D and Guilpain P: Adipose-derived mesenchymal stem cells in autoimmune disorders: State of the art and perspectives for systemic sclerosis. Clin Rev Allergy Immunol 52: 234-259, 2017.

15. Liu T, Mu H, Shen Z, Song Z, Chen X and Wang Y: Autologous adipose tissue-derived mesenchymal stem cells are involved in rat liver regeneration following repeat partial hepatectomy. Mol Med Rep 13: 2053-2059, 2016.

16. Kizilay Mancini O, Shum-Tim D, Stochaj U, Correa JA and Colmegna I: Age, atherosclerosis and type 2 diabetes reduce human mesenchymal stromal cell-mediated T-cell suppression. Stem Cell Res Ther 6: 140, 2015.

17. Wang YL, Li G, Zou XF, Chen XB, Liu T and Shen ZY: Effect of autologous adipose-derived stem cells in renal cold ischemia and reperfusion injury. Transplant Proc 45: 3198-3202, 2013.

18. Liu T, Zhang Y, Shen Z, Song Z, Zou X, Chen X, Chen L and Wang Y: Immunomodulatory effects of OX40Ig gene-modified adipose tissue-derived mesenchymal stem cells in rat kidney transplantation. Int J Mol Med 39: 144-152, 2017.

19. Livak KJ and Schmittgen TD: Analysis of relative gene expression data using real-time quantitative PCR and the 2(-Delta Delta C(T)) method. Methods 25: 402-408, 2001.

20. Wu YE, Du ZR, Cai YM, Peng WG, Zheng GZ, Zheng GL, Wu LB and Li K: Effective expansion of forkhead box $\mathrm{P}^{+}$regulatory T cells via early secreted antigenic target 6 and antigen 85 complex B from Mycobacterium tuberculosis. Mol Med Rep 11: 3134-3142, 2015

21. Zhang Z, Lin H, Shi M, Xu R, Fu J, Lv J, Chen L, Lv S, Li Y, Yu S, et al: Human umbilical cord mesenchymal stem cells improve liver function and ascites in decompensated liver cirrhosis patients. J Gastroenterol Hepatol 27 (Suppl 2): S112-S120, 2012.

22. Choudhery MS, Badowski M, Muise A, Pierce J and Harris DT: Donor age negatively impacts adipose tissue-derived mesenchymal stem cell expansion and differentiation. J Transl Med 12: 8, 2014.

23. Harasymiak-Krzyżanowska I, Niedojadło A, Karwat J, Kotuła L, Gil-Kulik P, Sawiuk M and Kocki J: Adipose tissue-derived stem cells show considerable promise for regenerative medicine applications. Cell Mol Biol Lett 18: 479-493, 2013.

24. Schneider S, Unger M, van Griensven M and Balmayor ER: Adipose-derived mesenchymal stem cells from liposuction and resected fat are feasible sources for regenerative medicine. Eur J Med Res 22: 17, 2017.

25. Efimenko A, Dzhoyashvili N, Kalinina N, Kochegura T, Akchurin R, Tkachuk V and Parfyonova Y: Adipose-derived mesenchymal stromal cells from aged patients with coronary artery disease keep mesenchymal stromal cell properties but exhibit characteristics of aging and have impaired angiogenic potential. Stem Cells Transl Med 3: 32-41, 2014.

26. Wu W, Niklason L and Steinbacher DM: The effect of age on human adipose-derived stem cells. Plast Reconstr Surg 131: 27-37, 2013.

27. Yarygin KN, Lupatov AY and Kholodenko IV: Cell-based therapies of liver diseases: Age-related challenges. Clin Interv Aging 10: 1909-1924, 2015.

28. Cai A, Qiu R, Li L, Zheng D, Dong Y, Yu D, Huang Y, Rao S, Zhou Y and Mai W: Atorvastatin treatment of rats with ischemia-reperfusion injury improves adipose-derivedmesenchymal stem cell migration and survival via the SDF- $1 \alpha / \mathrm{CXCR}-4$ axis. PLoS One 8: e79100, 2013.

29. Lai SL, Cheah SC, Wong PF, Noor SM and Mustafa MR: In vitro and in vivo anti-angiogenic activities of Panduratin A. PLoS One 7: e38103, 2012.

30. Ong WK and Sugii S: Adipose-derived stem cells: Fatty potentials for therapy. Int J Biochem Cell Biol 45: 1083-1086, 2013.

31. Kornicka K, Marycz K, Tomaszewski KA, Marędziak M and Śmieszek A: The effect of age on osteogenic and adipogenic differentiation potential of human adipose derived stromal stem cells (hASCs) and the impact of stress factors in the course of the differentiation process. Oxid Med Cell Longev 2015: 309169, 2015.

32. Beane OS, Fonseca VC, Cooper LL, Koren G and Darling EM: Impact of aging on the regenerative properties of bone marrow-, muscle-, and adipose-derived mesenchymal stem/stromal cells. PLoS One 9: e115963, 2014. 
33. Sethe S, Scutt A and Stolzing A: Aging of mesenchymal stem cells. Ageing Res Rev 5: 91-116, 2006.

34. Owen A and Newsome PN: Mesenchymal stromal cell therapy in liver disease: Opportunities and lessons to be learnt?. Am J Physiol Gastrointest Liver Physiol 309: G791-G800, 2015.

35. Petrou P, Gothelf Y, Argov Z, Gotkine M, Levy YS, Kassis I, Vaknin-Dembinsky A, Ben-Hur T, Offen D, Abramsky O, et al: Safety and clinical effects of mesenchymal stem cells secreting neurotrophic factor transplantation in patients with amyotrophic lateral sclerosis: Results of phase $1 / 2$ and 2 a clinical trials. JAMA Neurol 73: 337-344, 2016
36. Mizuno M, Katano H, Otabe K, Komori K, Matsumoto Y, Fujii S, Ozeki N, Tsuji K, Koga H, Muneta T, et al: Platelet-derived growth factor (PDGF)-AA/AB in human serum are potential indicators of the proliferative capacity of human synovial mesenchymal stem cells. Stem Cell Res Ther 6: 243, 2015.

37. Kavanagh DP, Suresh S, Newsome PN, Frampton J and Kalia N: Pretreatment of mesenchymal stem cells manipulates their vasculoprotective potential while not altering their homing within the injured gut. Stem Cells 33: 2785-2797, 2015. 